\title{
Higgs boson decay into 2 photons in the type II Seesaw model
}

\author{
A. Arhrib, ${ }^{a, b}$ R. Benbrik, ${ }^{b, c, d}$ M. Chabab, ${ }^{b}$ G. Moultaka ${ }^{e, f}$ and L. Rahili ${ }^{b}$ \\ ${ }^{a}$ Département de Mathématiques, Faculté des Sciences et Techniques, \\ Tanger, Morocco \\ ${ }^{b}$ Laboratoire de Physique des Hautes Energies et Astrophysique, \\ Université Cadi-Ayyad, FSSM, Marrakech, Morocco \\ ${ }^{c}$ Faculté Polydisciplinaire, Université Cadi Ayyad, \\ Sidi Bouzid, Safi-Morocco \\ ${ }^{d}$ Instituto de Fisica de Cantabria (CSIC-UC), \\ Santander, Spain \\ ${ }^{e}$ Université Montpellier 2, Laboratoire Charles Coulomb UMR 5221, \\ F-34095 Montpellier, France \\ ${ }^{f}$ CNRS, Laboratoire Charles Coulomb UMR 5221, \\ F-34095 Montpellier, France \\ E-mail: aarhrib@ictp.it, rbenbrik@ictp.it, m_chabab@yahoo.fr, \\ gilbert.moultaka@um2.fr, rahililarbi@gmail.com
}

ABStRaCt: We study the two photon decay channel of the Standard Model-like component of the CP-even Higgs bosons present in the type II Seesaw Model. The corresponding cross-section is found to be significantly enhanced in parts of the parameter space, due to the (doubly-)charged Higgs bosons' $\left(H^{ \pm \pm}\right) H^{ \pm}$virtual contributions, while all the other Higgs decay channels remain Standard Model(SM)-like. In other parts of the parameter space $H^{ \pm \pm}$(and $H^{ \pm}$) interfere destructively, reducing the two photon branching ratio tremendously below the SM prediction. Such properties allow to account for any excess such as the one reported by ATLAS/CMS at $\approx 125 \mathrm{GeV}$ if confirmed by future data; if not, for the fact that a SM-like Higgs exclusion in the diphoton channel around $114-115 \mathrm{GeV}$ as reported by ATLAS, does not contradict a SM-like Higgs at LEP(!), and at any rate, for the fact that ATLAS/CMS exclusion limits put stringent lower bounds on the $H^{ \pm \pm}$mass, particularly in the parameter space regions where the direct limits from same-sign leptonic decays of $H^{ \pm \pm}$do not apply.

Keywords: Higgs Physics, Beyond Standard Model 


\section{Contents}

1 Introduction 1

2 The DTHM model 3

$3 \mathcal{H} \rightarrow \gamma \gamma$

4 Theoretical and experimental constraints, numerics and discussions $\quad 8$

4.1 DTHM parameter scans and theoretical constraints 9

4.2 Experimental constraints 9

$\begin{array}{lll}4.3 & \text { Numerical results } & 10\end{array}$

$\begin{array}{llr}5 & \text { Conclusions } & 14\end{array}$

\section{Introduction}

The LHC running at $7 \mathrm{TeV}$ center of mass energy is accumulating more and more data. The ATLAS and CMS experiments have already probed the Higgs boson in the mass range 110- $600 \mathrm{GeV}$, and excluded a Standard Model (SM) Higgs in the range $141-476 \mathrm{GeV}$ at the $95 \%$ C.L. through a combined analysis of all decay channels and up to $\sim 2.3 \mathrm{fb}^{-1}$ integrated luminosity per experiment, [1]. Very recently, the analyses of $4.9 \mathrm{fb}^{-1}$ datasets for the combined channels made separately by ATLAS and by CMS, have narrowed further down the mass window for a light SM Higgs, excluding respectively the mass ranges 131$453 \mathrm{GeV}$ (apart from the range $237-251 \mathrm{GeV}$ ), [2], and $127-600 \mathrm{GeV}$ [3] at the $95 \%$ C.L. More interestingly, both experiments exclude 1 to 2-3 times the SM diphoton cross-section at the $95 \%$ C.L. in most of the mass range $110-130 \mathrm{GeV}$, and report an excess of events around $123-127 \mathrm{GeV}$ in the diphoton channel (as well as, but with lower statistical significance, in the $W W^{*}$ and $Z Z^{*}$ channels), corresponding to an exclusion of 3 and 4 times the SM cross-section respectively for CMS [4] and ATLAS [5]. Furthermore, they exclude a SM Higgs in small, though different, portions of this mass range, $114-115 \mathrm{GeV}$ for ATLAS and $127-131 \mathrm{GeV}$ for CMS, at the $95 \%$ C.L.

Notwithstanding the very exciting perspective of more data to come during the next LHC run, one remains for the time being free to interpret the present results as either pointing towards a SM Higgs around $125 \mathrm{GeV}$, or to a non-SM Higgs around $125 \mathrm{GeV}$ in excess of a few factors in the diphoton channel, or to behold that these results are still compatible with statistical fluctuations.

The main purpose of the present paper is not to show that the model we consider can account for a Higgs with mass $\approx 125 \mathrm{GeV}$, although it can do so as will become apparent in the sequel. Our aim will be rather to consider more globally how the recent 
experimental exclusion limits can constrain the peculiar features we will describe of the SM-like component of the model.

Although ATLAS/CMS exclusion limits assume SM-like branching ratios for all search channels, they can also be used in case the branching ratio of only the diphoton decay channel, $\operatorname{Br}(H \rightarrow \gamma \gamma)$, differs significantly from its $\mathrm{SM}$ value. This is due to the tininess of this branching ratio $\left(\lesssim 2 \times 10^{-3}\right)$, so that if enhanced even by more than an order of magnitude, due to the effects of some non-standard physics, all the other branching ratios would remain essentially unaffected. Thus, the present SM-like exclusion limits for the individual channels could still be directly applied. Furthermore, if this non-standard physics keeps the tree-level Higgs couplings to fermions and to $\mathrm{W}$ and $\mathrm{Z}$ gauge bosons very close to the SM ones, then obviously the corresponding channels will not lead to exclusions specific to this new physics. The diphoton channel becomes then of particular interest in this case and can already constrain parts of the parameter space of the new physics through the present exclusion limits in the Higgs mass range $114-130 \mathrm{GeV}$.

A natural setting for such a scenario is the Higgs sector of the so-called Type II Seesaw Model for neutrino mass generation [6-10]. This sector, containing two CP-even, one CPodd, one charged and one doubly-charged Higgs scalars, can be tested directly at the LHC, provided that the Higgs triplet mass scale $M_{\Delta}$ and the soft lepton-number violating mass parameter $\mu$ are of order or below the weak-scale [11-20]. Moreover, in most of the parameter space [and apart from an extremely narrow region of $\mu$ ], one of the two CP-even Higgs scalars is generically essentially SM-like and the other an almost decoupled triplet, irrespective of their relative masses, [20]. It follows that if all the Higgs sector of the model is accessible to the LHC, one expects a neutral Higgs state with cross-sections very close to the SM in all Higgs production and decay channels to leading electroweak order, except for the diphoton (and also $\gamma Z$ ) channel. Indeed, in the latter channel, loop effects of the other Higgs states can lead to substantial enhancements which can then be readily analyzed in the light of the experimental exclusion limits as argued above.

In this paper we will study quantitatively this issue. The main result is that the loop effects of the charged and in particular the doubly-charged Higgs states can either enhance the diphoton cross-section by several factors, or reduce it in some cases by several orders of magnitude essentially without affecting the other SM-like decay channels. This is consistent with the present experimental limits on these (doubly-)charged Higgs states masses and can be interpreted in several ways. It can account for an excess in the diphoton cross-section like the one observed by ATLAS/CMS. But it can also account for a deficit in the diphoton cross-section without affecting the other channels. The latter case could be particularly interesting for the 114-115 GeV SM Higgs mass range excluded by ATLAS, [provided one is willing to interpret the excess at $\approx 125 \mathrm{GeV}$ as statistical fluctuation]. Indeed, since the coupling of the Higgs to the $Z$ boson remains standard in our model, a possible LEP signal at $114-115 \mathrm{GeV}$ would remain perfectly compatible with the ATLAS exclusion!

The rest of the paper is organized as follows: in section 2 we briefly review some ingredients of the Higgs sector of the type II seesaw model, hereafter dubbed DTHM. In section 3 we calculate the branching ratio of $H \rightarrow \gamma \gamma$ in the context of DTHM and discuss its sensitivity to the parameters of the model.[The $\gamma Z$ channel can be treated along 
similar lines but will not be discussed in the present paper.] Section 4 is devoted to the theoretical and experimental constraints as well as to the numerical analysis for the physical observables. We conclude in section 5 .

\section{The DTHM model}

In [20] we have performed a detailed study of DTHM potential, derived the most general set of dynamical constraints on the parameters of the model at leading order and outlined the salient features of Higgs boson phenomenology at the colliders. These constraints delineate precisely the theoretically allowed parameter space domain that one should take into account in Higgs phenomenological analyses. We have also shown that in most of the parameter space the DTHM is similar to the SM except in the small $\mu$ regime where the doublet and triplet component of the Higgs could have a maximal mixing.

The scalar sector of the DTHM model consists of the standard Higgs doublet $H$ and a colorless Higgs triplet $\Delta$ with hypercharge $Y_{H}=1$ and $Y_{\Delta}=2$ respectively. Their matrix representation are given by:

$$
\Delta=\left(\begin{array}{cc}
\delta^{+} / \sqrt{2} & \delta^{++} \\
\delta^{0} & -\delta^{+} / \sqrt{2}
\end{array}\right) \quad \text { and } \quad H=\left(\begin{array}{c}
\phi^{+} \\
\phi^{0}
\end{array}\right)
$$

The most general $\mathrm{SU}(2)_{L} \times \mathrm{U}(1)_{Y}$ gauge invariant renormalizable Lagrangian in the scalar sector is $[12,20]$ :

$$
\mathcal{L}=\left(D_{\mu} H\right)^{\dagger}\left(D^{\mu} H\right)+\operatorname{Tr}\left(D_{\mu} \Delta\right)^{\dagger}\left(D^{\mu} \Delta\right)-V(H, \Delta)+\mathcal{L}_{\text {Yukawa }}
$$

where the potential $V(H, \Delta)$ is given by,

$$
\begin{aligned}
V= & -m_{H}^{2} H^{\dagger} H+\frac{\lambda}{4}\left(H^{\dagger} H\right)^{2}+M_{\Delta}^{2} \operatorname{Tr}\left(\Delta^{\dagger} \Delta\right)+\lambda_{1}\left(H^{\dagger} H\right) \operatorname{Tr}\left(\Delta^{\dagger} \Delta\right) \\
& +\lambda_{2}\left(\operatorname{Tr} \Delta^{\dagger} \Delta\right)^{2}+\lambda_{3} \operatorname{Tr}\left(\Delta^{\dagger} \Delta\right)^{2}+\lambda_{4} H^{\dagger} \Delta \Delta^{\dagger} H+\left[\mu\left(H^{T} i \tau_{2} \Delta^{\dagger} H\right)+h c\right]
\end{aligned}
$$

$\mathcal{L}_{\text {Yukawa }}$ contains all the SM Yukawa sector plus one extra term that provides, after spontaneous electroweak symmetry breaking (EWSB), a Majorana mass to neutrinos.

Once EWSB takes place, the Higgs doublet and triplet acquire vacuum expectation values

$$
\langle H\rangle=\frac{1}{\sqrt{2}}\left(\begin{array}{c}
0 \\
v_{d}
\end{array}\right), \quad\langle\Delta\rangle=\frac{1}{\sqrt{2}}\left(\begin{array}{cc}
0 & 0 \\
v_{t} & 0
\end{array}\right)
$$

inducing the $\mathrm{Z}$ and $\mathrm{W}$ masses

$$
M_{Z}^{2}=\frac{\left(g^{2}+g^{\prime 2}\right)\left(v_{d}^{2}+4 v_{t}^{2}\right)}{4} \quad, M_{W}^{2}=\frac{g^{2}\left(v_{d}^{2}+2 v_{t}^{2}\right)}{4}
$$

with $v^{2}=\left(v_{d}^{2}+4 v_{t}^{2}\right) \approx(246 \mathrm{GeV})^{2}$.

The DTHM is fully specified by seven independent parameters which we will take: $\lambda$, $\lambda_{i=1 \ldots 4}, \mu$ and $v_{t}$. These parameters respect a set of dynamical constraints originating from 
the potential, particularly perturbative unitarity and boundedness from below constraints

The model spectrum contains seven physical Higgs states: a pair of CP even states $\left(h^{0}, H^{0}\right)$, one CP odd Higgs boson $A$, one simply charged Higgs $H^{ \pm}$and one doubly charged state $H^{ \pm \pm}$. The squared masses of the neutral CP-even states and of the charged and doubly charged states are given in terms of the VEV's and the parameters of the potential as follows,

$$
\begin{aligned}
m_{h^{0}}^{2} & =\frac{1}{2}\left[A+C-\sqrt{(A-C)^{2}+4 B^{2}}\right] \\
m_{H^{0}}^{2} & =\frac{1}{2}\left[A+C+\sqrt{(A-C)^{2}+4 B^{2}}\right]
\end{aligned}
$$

with

$$
A=\frac{\lambda}{2} v_{d}^{2}, \quad B=v_{d}\left(-\sqrt{2} \mu+\left(\lambda_{1}+\lambda_{4}\right) v_{t}\right), \quad C=\frac{\sqrt{2} \mu v_{d}^{2}+4\left(\lambda_{2}+\lambda_{3}\right) v_{t}^{3}}{2 v_{t}}
$$

and

$$
\begin{aligned}
m_{H^{ \pm}}^{2} & =\frac{\left(v_{d}^{2}+2 v_{t}^{2}\right)\left[2 \sqrt{2} \mu-\lambda_{4} v_{t}\right]}{4 v_{t}} \\
m_{H^{ \pm \pm}}^{2} & =\frac{\sqrt{2} \mu v_{d}^{2}-\lambda_{4} v_{d}^{2} v_{t}-2 \lambda_{3} v_{t}^{3}}{2 v_{t}}
\end{aligned}
$$

For a recent and comprehensive study of the DTHM, in particular concerning the distinctive properties of the mixing angle between the neutral components of the doublet and triplet Higgs fields, we refer the reader to [20].

We close this section by stressing an important point which is seldom clearly stated in the literature. Recall first that the general rational justifying the name 'type II seesaw' assumes $\mu \sim M_{\Delta} \sim M_{\mathrm{GUT}}$ (or any other scale much larger than the electroweak scale). One then obtains naturally $v_{t} \ll v_{d}$, as a consequence of the electroweak symmetry breaking conditions, and thus naturally very small neutrino masses for Yukawa couplings of order 1. But then one has also $\mu \gg v_{t}$ and consequently a very heavy Higgs sector, largely out of the reach of the LHC, apart from the lightest state $h^{0}$, as can be seen from the above mass expressions; this leaves us at the electroweak scale with simply a SM Higgs sector. Put differently, a search for the DTHM Higgs states at the LHC entails small $\mu\left(\sim \mathcal{O}\left(v_{t}\right)\right)$ and thus implicitly questions the validity of the seesaw mechanism. Since we are interested in new physics visible at the LHC, we will take up the latter assumption of small $\mu$ in our phenomenological study, which can also have some theoretical justification related to spontaneous soft lepton-number violation.

\section{$3 \quad \mathcal{H} \rightarrow \gamma \gamma$}

The low SM Higgs mass region, $[110,140] \mathrm{GeV}$, is the most challenging for LHC searches. In this mass regime, the main search channel through the rare decay into a pair of photons can be complemented by the decay into $\tau^{+} \tau^{-}$and potentially the $b \bar{b}$ channel (particularly 

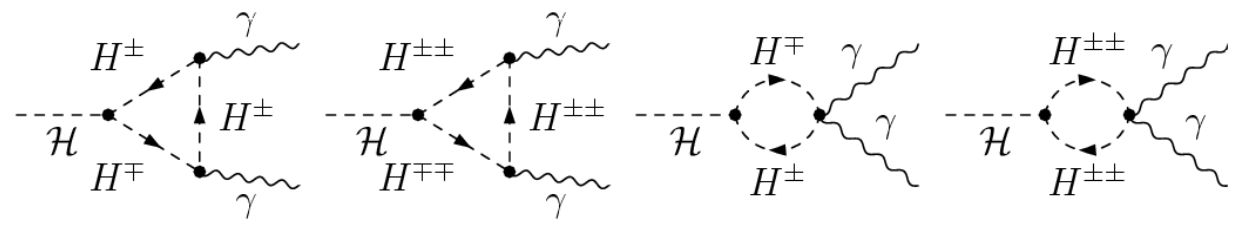

Figure 1. Singly and doubly charged Higgs bosons contributions to $\mathcal{H}\left(h^{0}, H^{0}\right) \rightarrow \gamma \gamma$ in the DTHM.

for the lower edge of the mass range and/or for supersymmetric Higgs searches), while the $W W^{*}, Z Z^{*}$ channels are already competitive in the upper edge $(130-140 \mathrm{GeV})$ of this mass range [1] the Higgs being produced mainly via gluon fusion [21, 22].

The theoretical predictions for the loop induced decays $H \rightarrow \gamma \gamma$ (and $H \rightarrow \gamma Z$ ) have been initiated since many years [23-25]. Several more recent studies have been carried out looking for large loop effects. Such large effects can be found in various extensions of the SM, such as the Minimal Supersymmetric Standard Model (MSSM) [26-29], the two Higgs Doublet Model [30-36], the Next-to-MSSM [37-39], the little Higgs models [40, 41], in models with a real triplet [42] and in Randall-Sundrum models [43]. To the best of our knowledge there is no $H \rightarrow \gamma \gamma$ study in the context of a triplet field with hypercharge $Y=2$, that is comprising charged and doubly-charged Higgs states.

We turn here to the study of the latter case explaining how these charged and doubly charged Higgs states of the DTHM could enhance or suppress the 2 photons decay rate. Furthermore, since one or the other of the two CP-even neutral Higgs bosons $h^{0}, H^{0}$ present in the DTHM can behave as a purely SM-like Higgs depending on the regime under consideration (see [20]), we will refer to the SM-like state generically as $\mathcal{H}$ in the following. It should be kept in mind, however, that when $\mathcal{H}=h^{0}$ all the other DTHM Higgs states are heavier than $\mathcal{H}$ while when $\mathcal{H}=H^{0}$ they are all generically lighter than $\mathcal{H}$, thereby leading possibly to a different phenomenological interpretation of the present experimental exclusion limits for $\mathcal{H} \rightarrow \gamma \gamma$ channel.

The decay $\mathcal{H} \rightarrow \gamma \gamma$ is mediated at 1-loop level by the virtual exchange of the SM fermions, the SM gauge bosons and the new charged Higgs states. Using the general results for spin- $1 / 2$, spin-1 and spin-0 contributions, [25] (see also [44-46]), one includes readily the extra contributions to the partial width which takes the following form,

$$
\begin{aligned}
\Gamma(\mathcal{H} \rightarrow \gamma \gamma)= & \frac{G_{F} \alpha^{2} M_{\mathcal{H}}^{3}}{128 \sqrt{2} \pi^{3}} \mid \sum_{f} N_{c} Q_{f}^{2} \tilde{g}_{\mathcal{H} f f} A_{1 / 2}^{\mathcal{H}}\left(\tau_{f}\right)+\tilde{g}_{\mathcal{H} W W} A_{1}^{\mathcal{H}}\left(\tau_{W}\right) \\
& +\tilde{g}_{\mathcal{H} H^{ \pm} H^{\mp}} A_{0}^{\mathcal{H}}\left(\tau_{H^{ \pm}}\right)+\left.4 \tilde{g}_{\mathcal{H} H^{ \pm \pm} H^{\mp \mp}} A_{0}^{\mathcal{H}}\left(\tau_{H^{ \pm \pm}}\right)\right|^{2}
\end{aligned}
$$

where the first two terms in the squared amplitude are the known SM contributions up to the difference in the couplings of $\mathcal{H}$ to up and down quarks and $W^{ \pm}$in the DTHM, when $\mathcal{H}$ is not purely SM-like. The relevant reduced couplings (relative to the SM ones) are summarized in table. 1 . In eq. (3.1) $N_{c}=3(1)$ for quarks (leptons), $Q_{f}$ is the electric charge of the SM fermion $f$. The scalar functions $A_{1 / 2}^{\mathcal{H}}$ for fermions and $A_{1}^{\mathcal{H}}$ for gauge bosons are known in the literature and will not be repeated here (for a review see for 
instance [46]). The last two terms correspond to the $H^{ \pm}$and $H^{ \pm \pm}$contributions whose Feynman diagrams are depicted in Fig 1. The structure of the $H^{ \pm}$and $H^{ \pm \pm}$contributions is the same except for the fact that the $H^{ \pm \pm}$contribution is enhanced by a relative factor four in the amplitude since $H^{ \pm \pm}$has an electric charge of \pm 2 units. The scalar function for spin- $0 A_{0}^{\mathcal{H}}$ is defined as

$$
A_{0}^{\mathcal{H}}(\tau)=-[\tau-f(\tau)] \tau^{-2}
$$

with $\tau_{i}=m_{\mathcal{H}}^{2} / 4 m_{i}^{2}\left(i=f, W, H^{ \pm}, H^{ \pm \pm}\right)$and the function $f(\tau)$ is given by

$$
f(\tau)= \begin{cases}\arcsin ^{2} \sqrt{\tau} & \tau \leq 1 \\ -\frac{1}{4}\left[\log \frac{1+\sqrt{1-\tau^{-1}}}{1-\sqrt{1-\tau^{-1}}}-i \pi\right]^{2} & \tau>1\end{cases}
$$

while the reduced DTHM trilinear couplings of $\mathcal{H}$ to $H^{ \pm}$and $H^{ \pm \pm}$are given by

$$
\begin{aligned}
\tilde{g}_{\mathcal{H} H^{++} H^{--}} & =-\frac{s_{W}}{e} \frac{m_{W}}{m_{H^{ \pm \pm}}^{2}} g_{\mathcal{H} H^{++} H^{--}} \\
\tilde{g}_{\mathcal{H} H^{+} H^{-}} & =-\frac{s_{W}}{e} \frac{m_{W}}{m_{H^{ \pm}}^{2}} g_{\mathcal{H} H^{+} H^{-}}
\end{aligned}
$$

with

$$
\begin{aligned}
g_{\mathcal{H} H^{++} H^{--}} & \approx-\bar{\epsilon} \lambda_{1} v_{d} \\
g_{\mathcal{H} H^{+} H^{-}} & \approx-\bar{\epsilon}\left(\lambda_{1}+\frac{\lambda_{4}}{2}\right) v_{d}
\end{aligned}
$$

The latter can be read off from the couplings of $h^{0}$,

$$
\begin{aligned}
g_{h^{0} H^{++} H^{--}}= & -\left\{2 \lambda_{2} v_{t} s_{\alpha}+\lambda_{1} v_{d} c_{\alpha}\right\} \\
g_{h^{0} H^{+} H^{-}}= & -\frac{1}{2}\left\{\left\{4 v_{t}\left(\lambda_{2}+\lambda_{3}\right) c_{\beta^{\prime}}^{2}+2 v_{t} \lambda_{1} s_{\beta^{\prime}}^{2}-\sqrt{2} \lambda_{4} v_{d} c_{\beta^{\prime}} s_{\beta^{\prime}}\right\} s_{\alpha}\right. \\
& \left.+\left\{\lambda v_{d} s_{\beta^{\prime}}^{2}+\left(2 \lambda_{1}+\lambda_{4}\right) v_{d} c_{\beta^{\prime}}^{2}+\left(4 \mu-\sqrt{2} \lambda_{4} v_{t}\right) c_{\beta^{\prime}} s_{\beta^{\prime}}\right\} c_{\alpha}\right\}
\end{aligned}
$$

in the limit where $h^{0}$ becomes a pure SM Higgs, i.e. when $s_{\alpha} \rightarrow 0$, or from the couplings of $H^{0}$, obtained simply from the above couplings by the substitutions

$$
\begin{aligned}
g_{H^{0} H^{++} H^{--}} & =g_{h^{0} H^{++} H^{--}}\left[c_{\alpha} \rightarrow-s_{\alpha}, s_{\alpha} \rightarrow c_{\alpha}\right] \\
g_{H^{0} H^{+} H^{-}} & =g_{h^{0} H^{+} H^{-}}\left[c_{\alpha} \rightarrow-s_{\alpha}, s_{\alpha} \rightarrow c_{\alpha}\right]
\end{aligned}
$$

in the limit where $H^{0}$ becomes a pure SM Higgs, i.e. when $c_{\alpha} \rightarrow 0$, taking also into account that $s_{\beta^{\prime}} \approx \sqrt{2} v_{t} / v_{d}$ with $v_{t} / v_{d} \ll 1$. [In the above equations $\alpha$ and $\beta^{\prime}$ stand for the mixing angles in the CP-even and charged Higgs sectors with the shorthand notations $s_{x}, c_{x}$ for $\cos x, \sin x$; In eqs. (3.6), (3.7) we have denoted by $\bar{\epsilon}$ the sign of $s_{\alpha}$ in the convention where $c_{\alpha}$ is always positive, which is defined as $\bar{\epsilon}=1$ for $\mathcal{H} \equiv h^{0}$ and $\bar{\epsilon}=\operatorname{sign}\left[\sqrt{2} \mu-\left(\lambda_{1}+\lambda_{4}\right) v_{t}\right]$ for $\mathcal{H} \equiv H^{0}$; see [20].] Obviously, in the limit where one of the two CP-even Higgs states is 


\begin{tabular}{|c|c|c|c|}
\hline $\mathcal{H}$ & $\tilde{g}_{\mathcal{H} \bar{u} u}$ & $\tilde{g}_{\mathcal{H} \bar{d} d}$ & $\tilde{g}_{\mathcal{H} W^{+} W^{-}}$ \\
\hline$h^{0}$ & $c_{\alpha} / c_{\beta^{\prime}}$ & $c_{\alpha} / c_{\beta^{\prime}}$ & $+e\left(c_{\alpha} v_{d}+2 s_{\alpha} v_{t}\right) /\left(2 s_{W} m_{W}\right)$ \\
$H^{0}$ & $-s_{\alpha} / c_{\beta^{\prime}}$ & $-s_{\alpha} / c_{\beta^{\prime}}$ & $-e\left(s_{\alpha} v_{d}-2 c_{\alpha} v_{t}\right) /\left(2 s_{W} m_{W}\right)$ \\
\hline
\end{tabular}

Table 1. The CP-even neutral Higgs couplings to fermions and gauge bosons in the DTHM relative to the SM Higgs couplings, $\alpha$ and $\beta^{\prime}$ denote the mixing angles respectively in the CP-even and charged Higgs sectors, $e$ is the electron charge, $m_{W}$ the $W$ gauge boson mass and $s_{W}$ the weak mixing angle.

SM-like, the other state behaves as a pure triplet $\Delta^{0}$ with suppressed couplings to $H^{ \pm}$and $H^{ \pm \pm}$given by $g_{\Delta^{0} H^{+} H^{-}} \approx\left(\lambda_{4} / \sqrt{2}-2\left(\lambda_{2}+\lambda_{3}\right)\right) v_{t}$ and $g_{\Delta^{0} H^{++} H^{--}} \approx-2 \lambda_{2} v_{t}$. Due to the smallness of $v_{t} / v_{d}$ the states $h^{0}, H^{0}$ are mutually essentially SM-like or essentially triplet, apart from a very tiny and fine-tuned region where they carry significant components of both. (see [20] for more details). We can thus safely consider that any experimental limit on the SM Higgs decay in two photons can be applied exclusively either to $h^{0}$ or to $H^{0}$, depending on whether we assume $\mathcal{H}$ to be the lightest or the heaviest among all the neutral and charged Higgs states of the DTHM. ${ }^{1}$

As a cross-check on our tools, an independent calculation using the FeynArts and FormCalc $[47,48]$ packages for which we provided a DTHM model file was also carried out and we found perfect agreement with eq. (3.1). Clearly the contribution of the $H^{ \pm \pm}$ and $H^{ \pm}$loops depends on the details of the scalar potential. The phase space function $A_{0}$ involves the scalar masses $m_{H^{ \pm}}$and $m_{H^{ \pm \pm}}$, while $g_{\mathcal{H} H^{+} H^{-}}$and $g_{\mathcal{H} H^{++} H^{--}}$are functions of several Higgs potential parameters. It is clear from eqs. (3.6), (3.7) that those couplings are not suppressed in the small $v_{t}$ and/or $\sin \alpha$ limit but have a contribution which is proportional to the vacuum expectation value of the doublet field and hence can be a source of large enhancement of $\mathcal{H} \rightarrow \gamma \gamma$ (and $\mathcal{H} \rightarrow \gamma Z$ ).

As well known, the decay width of $\mathcal{H} \rightarrow \gamma \gamma$ in the $\mathrm{SM}$ is dominated by the $\mathrm{W}$ loops which can also interfere destructively with the subdominant top contribution. In the DTHM, the signs of the couplings $g_{\mathcal{H} H^{+} H^{-}}$and $g_{\mathcal{H} H^{++} H^{--}}$, and thus those of the $H^{ \pm}$ and $H^{ \pm \pm}$contributions to $\Gamma(\mathcal{H} \rightarrow \gamma \gamma)$, are fixed respectively by the signs of $2 \lambda_{1}+\lambda_{4}$ and $\lambda_{1}$, eqs. (3.4), (3.5), (3.6), (3.7). However, the combined perturbative unitarity and potential boundedness from below (BFB) constraints derived in [20] confine $\lambda_{1}, \lambda_{4}$ to small regions. For instance, in the case of vanishing $\lambda_{2,3}, \lambda_{1}$ is forced to be positive while $\lambda_{4}$ can have either signs but still with bounded values of $\left|\lambda_{4}\right|$ and $\left|2 \lambda_{1}+\lambda_{4}\right|$. Moreover, since we are considering scenarios where $\mu \sim \mathcal{O}\left(v_{t}\right)$, negative values of $\lambda_{4}$ can be favored by the experimental bounds on the (doubly)charged Higgs masses, eqs. (2.8), (2.9). For definiteness we stick in the following to $\lambda_{1}>0$, although the sign of $\lambda_{1}$ can be relaxed if $\lambda_{2,3}$ are non-vanishing. Also in the considered mass range for $\mathcal{H}, H^{ \pm}$and $H^{ \pm \pm}$the function $A_{0}^{\mathcal{H}}(\tau)$ is real-valued and takes positive values in the range $0.3-1$. An increasing value of $\lambda_{1}$ will

\footnotetext{
${ }^{1}$ The above mentioned tiny region with mixed states can also be treated, provided one includes properly the contribution of both $h^{0}$ and $H^{0}$, which are in this case almost degenerate in mass as well as with all the other Higgs masses of the DTHM.
} 
thus lead to contributions of $H^{ \pm}$and $H^{ \pm \pm}$that are constructive among each other but destructive with respect to the sum of $W$ boson and top quark contributions. [Recall that $\mathcal{R} e A_{1}^{\mathcal{H}}(\tau)$ takes negative values in the range -12 to -7 .] As we will see in the next section, this can either reduce tremendously the branching ratio into diphotons, or increase it by an amount that can be already constrained by the present ATLAS/CMS results.

\section{Theoretical and experimental constraints, numerics and discussions}

In order to infer limits on the parameters of our model from the experimental searches, we consider the ratio $\sigma^{\gamma \gamma} / \sigma_{S M}^{\gamma \gamma}$ that has been constrained by the recent ATLAS and CMS limits and compare it to the quantity

$$
R_{\gamma \gamma}(\mathcal{H})=\frac{(\Gamma(\mathcal{H} \rightarrow g g) \times \operatorname{Br}(\mathcal{H} \rightarrow \gamma \gamma))^{D T H M}}{(\Gamma(\mathcal{H} \rightarrow g g) \times \operatorname{Br}(\mathcal{H} \rightarrow \gamma \gamma))^{S M}}
$$

Note that the experimental limits on $\sigma^{\gamma \gamma} / \sigma_{S M}^{\gamma \gamma}$ assume all Higgs production channels, as well as SM-like Higgs decay branching ratios, taking into account the known QCD and EW corrections and uncertainties in the proton-proton collision, [49], some of which cancel out in the ratio. In contrast, the ratio $R_{\gamma \gamma}$ obviously concerns only the leading parton level gluon fusion Higgs production contribution, and assumes the narrow width approximation. We wish to comment here briefly on the approximations involved when identifying these two ratios, keeping in mind that in our present exploratory study we do not aim at a high precision analysis that would require a more quantitative estimate of the effects of these approximations. Strictly speaking, in the narrow width approximation the cross-section $\sigma(g g \rightarrow \mathcal{H} \rightarrow \gamma \gamma)$ is related to $\operatorname{Br}(\mathcal{H} \rightarrow g g) \times \operatorname{Br}(\mathcal{H} \rightarrow \gamma \gamma)$ up to a phase space factor, [50]. The ratio $r=\sigma(g g \rightarrow \mathcal{H} \rightarrow \gamma \gamma)^{D T H M} / \sigma(g g \rightarrow \mathcal{H} \rightarrow \gamma \gamma)^{S M}$ of the DTHM to SM values of this cross-section reduces then to $r=\Gamma(\mathcal{H} \rightarrow \text { all })^{S M} / \Gamma(\mathcal{H} \rightarrow \text { all })^{D T H M} \times R_{\gamma \gamma}(\mathcal{H})$. Taking into account the fact that in the SM-like Higgs regime of DTHM, the branching ratios of all Higgs decay channels are the same as in the SM, except for $\mathcal{H} \rightarrow \gamma \gamma$ (and $\mathcal{H} \rightarrow \gamma Z$ ) where they can significantly differ but remain very small compared to the other decay channels, one expects $\Gamma(\mathcal{H} \rightarrow \text { all })^{S M} / \Gamma(\mathcal{H} \rightarrow \text { all })^{D T H M} \approx 1 . R_{\gamma \gamma}(\mathcal{H})$ is thus a very good estimate of $r$ in this regime.

Nonetheless, using the experimental limits on $\sigma^{\gamma \gamma} / \sigma_{S M}^{\gamma \gamma}$ to constrain $R_{\gamma \gamma}(\mathcal{H})$ entails in principle subtracting from the former the subleading channels; mainly the $W$-boson fusion process which is of order $5-8 \%$ of the gluon fusion for $m_{\mathcal{H}}$ in the range $115-130 \mathrm{GeV}$, [49], and taking into account possible differences in the experimental analysis efficiency between these two channels. In the pure SM-like regime, there can be some instances where all details of the production channels cancel out in the ratio $\sigma^{\gamma \gamma} / \sigma_{S M}^{\gamma \gamma}$ due to a complete factorization of the Higgs branching ratio into two photons that contains all the new DTHM effects. In this case $\sigma^{\gamma \gamma} / \sigma_{S M}^{\gamma \gamma}$ becomes insensitive to the production channels and one retrieves essentially $R_{\gamma \gamma}(\mathcal{H})\left(\approx \operatorname{Br}(\mathcal{H} \rightarrow \gamma \gamma)^{D T H M} / \operatorname{Br}(\mathcal{H} \rightarrow \gamma \gamma)^{S M}\right)$. However, this cancellation can be model-dependent and does not necessarily occur when the $W$-boson fusion process is included. Indeed in this case the relevant parton-level process, $q q \rightarrow q q \mathcal{H} \rightarrow q q \gamma \gamma$, with the requirement of two hard jets in the final state, does not proceed via the exchange of a 
single intermediate unstable particle, due to the $W W$ intermediate states. The validity of the factorization of $\operatorname{Br}(\mathcal{H} \rightarrow \gamma \gamma)$ through the narrow width approximation requires here, and in contrast with the $g g \rightarrow \mathcal{H} \rightarrow \gamma \gamma$ case, that the variation of $\Gamma(\mathcal{H} \rightarrow \gamma \gamma)$ as a function of $m_{\mathcal{H}}$ should remain much larger than the Higgs total width itself, [50]. We find that the latter requirement is far from being satisfied in our case. In particular, we checked that in the experimentally interesting Higgs mass range around $125 \mathrm{GeV}, \Gamma(\mathcal{H} \rightarrow \gamma \gamma)$ becomes a very flat function of $m_{\mathcal{H}}$ leading to a variation at least an order of magnitude smaller than $\Gamma(\mathcal{H} \rightarrow$ all $)$. This invalidates the above mentioned factorization so that the ratio $\sigma^{\gamma \gamma} / \sigma_{S M}^{\gamma \gamma}$ remains sensitive to the initial state and does not reduce trivially to $R_{\gamma \gamma}$. One should thus keep in mind the corresonding approximations when using $R_{\gamma \gamma}$ instead of the experimentally constrained quantity. Of course the PDF uncertainties as well as the initial state leading QCD corrections drop out in the ratios $r$ and $R_{\gamma \gamma}$, even in the regime where $\mathcal{H}$ is not purely SM-like. However, QCD corrections to the fermion loop contribution to $\mathcal{H} \rightarrow \gamma \gamma$ should be in principle included as they can somewhat affect the interference pattern between the standard model and the (doubly)charged Higgs states contributions to be discussed in section 4.3 .

\subsection{DTHM parameter scans and theoretical constraints}

All the Higgs mass spectrum of the model is fixed in terms of $\lambda, \lambda_{1,2,3,4}, v_{t}$ and $\mu$ which we will take as input parameters, [20]. As one can see from eq. (2.3) $\lambda_{2}$ and $\lambda_{3}$ enter only the purely triplet sector. Since we focus here on the SM-like (doublet) component, their contributions will always be suppressed by the triplet VEV value and can be safely neglected as compared to the contributions of $\lambda_{1}$ and $\lambda_{4}$ which enter the game associated with the doublet VEV, eqs. (3.8)-(3.11). Taking into account the previous comments, $\lambda_{2,3}$ will be fixed and we perform a scan over the other parameter as follows:

$$
\begin{aligned}
& v_{t}=1 \mathrm{GeV}, \\
& \lambda=0.45 \sim 1, \\
& 0<\lambda_{1}<10, \\
& \lambda_{3}=2 \lambda_{2}=0.2 \text {, } \\
& 0.2 \mathrm{GeV}<\mu<20 \mathrm{GeV} \text {, } \\
& -5<\lambda_{4}<3
\end{aligned}
$$

The chosen range for $\lambda$ values ensures a light SM-like Higgs state and the scanned domain of the $\lambda_{i}$ 's is consistent with the perturbative unitarity and BFB bounds mentioned earlier.

\subsection{Experimental constraints}

Here we will discuss the experimental constraints on the triplet vev as well as on the scalar particles of the DTHM. In the above scan, the triplet vev has been taken equal to $1 \mathrm{GeV}$ in order to satisfy the constraint from the $\rho$ parameter [51] for which the tree-level extra contribution $\delta \rho$ should not exceed the current limits from precision measurements: $|\delta \rho| \lesssim 0.001$

Nowadays, the doubly charged Higgs boson is subject to many experimental searches. Due to its spectacular signature from $H^{ \pm \pm} \rightarrow l^{ \pm} l^{ \pm}$, the doubly charged Higgs has been searched by many experiments such as LEP, Tevatron and LHC. At the Tevatron, D $\emptyset[52$, 53] and CDF [54, 55] excluded a doubly charged Higgs with a mass in the range $100 \rightarrow$ $150 \mathrm{GeV}$. Recently, CMS also performed with $1 \mathrm{fb}^{-1}$ luminosity a search for doubly charged 
Higgs decaying to a pair of leptons, setting a lower mass limit of $313 \mathrm{GeV}$ from $H^{ \pm \pm} \rightarrow$ $\mu^{ \pm} \mu^{ \pm}, e^{ \pm} e^{ \pm}, \mu^{ \pm} e^{ \pm}[56]$. The limit is lower if we consider the other decay channel with one electron or more $[56,57]$.

We stress that all those bounds assume a $100 \%$ branching ratio for $H^{ \pm \pm} \rightarrow l^{ \pm} l^{ \pm}$ decay, while in realistic cases one can easily find scenarios where this decay channel is suppressed whith respect to $H^{ \pm \pm} \rightarrow W^{ \pm} W^{ \pm(*)}[11,12,58,59]$ which could invalidate partially the CDF, D $\emptyset$, CMS and ATLAS limits. In our scenario with $v_{t} \lesssim 1 \mathrm{GeV}$ we estimated that the decay channel $H^{ \pm \pm} \rightarrow W^{ \pm} W^{ \pm *}$ can still overwhelm the two-lepton channel for $m_{H^{ \pm \pm}}$down to $\approx 110 \mathrm{GeV}$. It has been shown in [60] that (cascade) channels such as $H^{ \pm \pm} \rightarrow H^{ \pm} W^{ \pm *} \rightarrow H^{0} W^{ \pm *} W^{ \pm *}$ can also compete strongly with $H^{ \pm \pm} \rightarrow l^{ \pm} l^{ \pm}$, lowering the $m_{H^{ \pm \pm}}$mass bound to $100 \mathrm{GeV}$. We will take $m_{H^{ \pm \pm}} \approx 110 \mathrm{GeV}$ as a nominal lower bound in our numerical analysis.

In the case of charged Higgs boson, if it decays dominantly to leptons or to light quarks $c s$ (for small $v_{t}$ ) we can apply the LEP mass lower bounds that are of the order of $80 \mathrm{GeV}[61,62]$. For large $v_{t}$, i.e. much larger than the neutrino masses but still well below the electroweak scale, the dominant decay is either $H^{+} \rightarrow t \bar{b}$ or one of the bosonic decays $H^{+} \rightarrow W^{+} Z, H^{+} \rightarrow W^{+} h^{0} / W^{+} A^{0}$. For the first two decay modes there has been no explicit search neither at LEP nor at the Tevatron, while for the $H^{+} \rightarrow W^{+} A^{0}$ decay (and possibly for $H^{+} \rightarrow W^{+} h^{0} /$ if $h^{0}$ decays similarly to $A^{0}$ ), one can use the LEPII search performed in the framework of two Higgs doublet models. In this case the charged Higgs mass limit is again of the order of $80 \mathrm{GeV}$ [62].

\subsection{Numerical results}

In the subsequent numerical discussion we use the following input parameters: $G_{F}=$ $1.166 \times 10^{-5} \mathrm{GeV}^{-2}, \alpha^{-1} \approx 128, m_{Z}=91.1875 \mathrm{G} \mathrm{eV}, m_{W}=80.45 \mathrm{GeV}$ and $m_{t}=173 \mathrm{GeV}$. We also compute the total width of the Higgs boson taking into account leading order QCD corrections as given in [63] as well as the off-shell decays $\mathcal{H} \rightarrow W W^{*}$ and $\mathcal{H} \rightarrow Z Z^{*}$ [64, 65].

We show in figure 2 the branching ratio for the CP-even Higgs bosons decays into two photons as a function of $\lambda_{1}$, illustrated for several values of $\lambda_{4}$ and $\lambda=0.45, v_{t}=1 \mathrm{GeV}$. In the left panel we take $\mu=1 \mathrm{GeV}$, implying that the lightest CP-even state $h^{0}$ carries $99 \%$ of the SM-like Higgs component, with an essentially fixed mass $m_{h^{0}} \approx 114-115 \mathrm{GeV}$ over the full range of values considered for $\lambda_{1}$ and $\lambda_{4}$. In the right panel, where $\mu=0.3 \mathrm{GeV}$, the heaviest CP-even state $H^{0}$ carries most of the SM-like Higgs component $[\sim 90 \%$ for $\left.\lambda_{1} \lesssim 3\right]$ with a mass more sensitive to the $\lambda_{1}$ and $\lambda_{4}$ couplings, $m_{H^{0}} \approx 115-123 \mathrm{GeV} .^{2}$

As can be seen from the plots, $\operatorname{Br}(\mathcal{H} \rightarrow \gamma \gamma)$ is very close to the $\mathrm{SM}$ prediction $[\approx$ $2 \times 10^{-3}$ ] for small values of $\lambda_{1}$, irrespective of the values of $\lambda_{4}$. Indeed in this region the diphoton decay is dominated by the SM contributions, the $H^{ \pm \pm}$contribution being shutdown for vanishing $\lambda_{1}$, cf. eq. (3.6), while the sensitivity to $\lambda_{4}$ in the $H^{ \pm}$contribution, eqs. (3.5), (3.7), is suppressed by a large $m_{H^{ \pm}}$mass, $m_{H^{ \pm}} \approx 164-237 \mathrm{GeV}$ for $-1<\lambda_{4}<$ 1. Increasing $\lambda_{1}$ (for fixed $\lambda_{4}$ ) enhances the $g_{\mathcal{H} H^{ \pm} H^{\mp}}$ and $g_{\mathcal{H} H^{ \pm \pm} H^{\mp \mp}}$ couplings. The

\footnotetext{
${ }^{2}$ In the latter case one has to be cautious in the range $\lambda_{1} \lesssim 4-10$ where $H^{0}$ carries only $75-85 \%$ of the SM-like component. The effects of the lighter state $h^{0}$ with a reduced coupling to the SM particles and a mass between $102-110 \mathrm{GeV}$, should then be included in the estimate of the overall diphoton cross-section.
} 

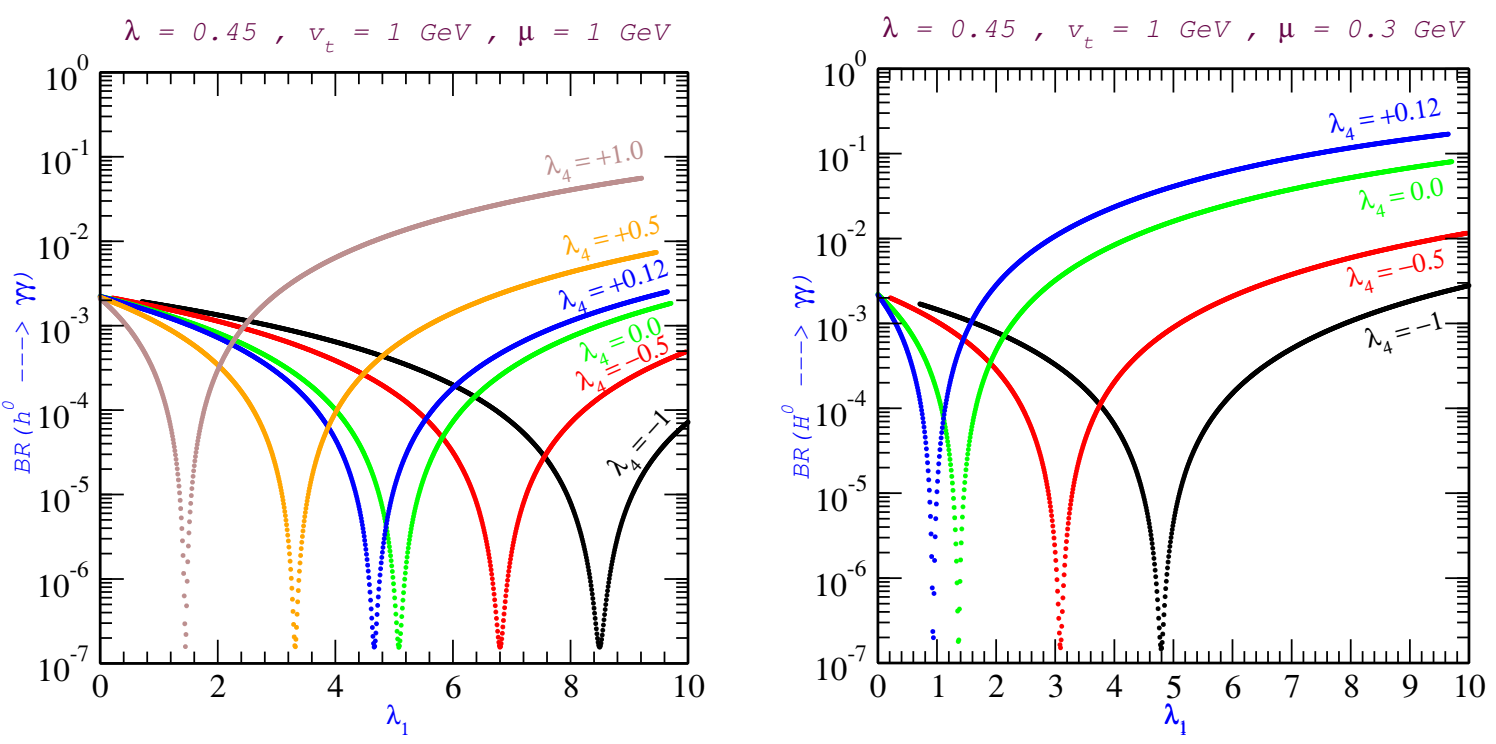

Figure 2. The branching ratios for $\mathcal{H} \rightarrow \gamma \gamma$ as a function of $\lambda_{1}$ for various values of $\lambda_{4}$ with $\lambda=0.45, \lambda_{3}=2 \lambda_{2}=0.2$ and $v_{t}=1 \mathrm{GeV}$; left panel: $\mu=1 \mathrm{GeV}, h^{0}$ is SM-like and $m_{h^{0}}=114-$ $115 \mathrm{GeV}$; right panel: $\mu=0.3 \mathrm{GeV}, H^{0}$ is SM-like and $m_{H^{0}}=115-123 \mathrm{GeV}$.

destructive interference, already noted in section 3, between the SM loop contributions and those of $H^{ \pm}$and $H^{ \pm \pm}$becomes then more and more pronounced. The leading DTHM effect is mainly from the $H^{ \pm \pm}$contribution, the latter being enhanced with respect to $H^{ \pm}$ by a factor 4 due to the doubled electric charge, but also due to a smaller mass than the latter in some parts of the parameter space, $m_{H^{ \pm \pm}} \approx 110-266 \mathrm{GeV}$. It is easy to see from eqs. (3.1), (3.4)-(3.7) that the amplitude for $\mathcal{H} \rightarrow \gamma \gamma$ is essentially linear in $\lambda_{1}$, since $m_{H^{ \pm}}$ and $m_{H^{ \pm \pm}}$, eqs. (2.8), (2.9), do not depend on $\lambda_{1}$ while the dependence on this coupling through $m_{\mathcal{H}}$ is screened by the mild behavior of the scalar functions $A_{0,1 / 2,1}^{\mathcal{H}}$. Furthermore, the latter functions remain real-valued in the considered domain of Higgs masses. There exit thus necessarily values of $\lambda_{1}$ where the effect of the destructive interference is maximized leading to a tremendous reduction of $\Gamma(\mathcal{H} \rightarrow \gamma \gamma)$. Since all the other decay channels remain SM-like, the same reduction occurs for $\operatorname{Br}(\mathcal{H} \rightarrow \gamma \gamma)$. The different dips seen in figure 2 are due to such a severe cancellation between SM loops and $H^{ \pm}$and $H^{ \pm \pm}$loops, and they occur for $\lambda_{1}$ values within the allowed unitarity \& BFB regions. Increasing $\lambda_{1}$ beyond the dip values, the contributions of $H^{ \pm \pm}$and $H^{ \pm}$become bigger than the SM contributions and eventually come to largely dominate for sufficiently large $\lambda_{1}$. There is however another interesting effect when $\lambda_{4}$ increases. Of course the locations of the dips depend also on the values of $\lambda_{4}$, moving them to lower values of $\lambda_{1}$ for larger $\lambda_{4}$. Thus, for larger $\lambda_{4}$, there is place, within the considered range of $\lambda_{1}$, for a significant increase of $\operatorname{Br}(\mathcal{H} \rightarrow \gamma \gamma)$ by even more than one order of magnitude with respect to the SM prediction. This spectacular enhancement is due to the fact that larger $\lambda_{4}$ leads to smaller $H^{ \pm \pm}$and $H^{ \pm}$which can efficiently boost the reduced couplings that scale like the inverse second power of these masses. For instance varying $\lambda_{4}$ between -1 and 1 in the left panel case, decreases $H^{ \pm \pm}$ from 266 to $110 \mathrm{GeV}$, while varying it from -1 to 0 in the right panel case decreases $H^{ \pm \pm}$ 

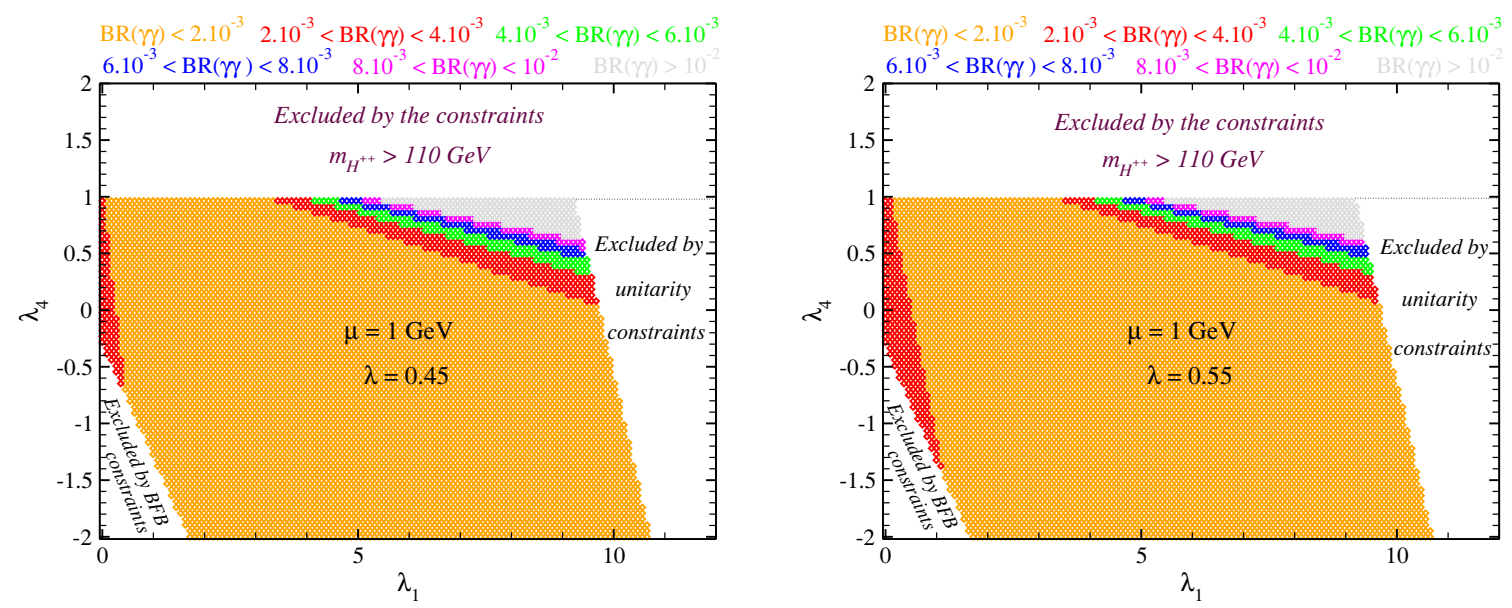

Figure 3. Scatter plot in the $\left(\lambda_{1}, \lambda_{4}\right)$ plane showing the branching ratios for $\mathcal{H} \rightarrow \gamma \gamma$. In both panels the SM-like Higgs is $h^{0}$, with $\lambda=0.45, m_{h^{0}} \approx 115 \mathrm{GeV}$ (left panel) and $\lambda=0.55, m_{h^{0}} \approx$ $127 \mathrm{GeV}$ (right panel); $\lambda_{3}=2 \lambda_{2}=0.2$ and $\mu=v_{t}=1 \mathrm{GeV}$.

from 205 to $112 \mathrm{GeV}$. In both cases we see $\operatorname{Br}(\mathcal{H} \rightarrow \gamma \gamma)$ rising by 2 orders of magnitude with respect to the SM value.

In figure 3 we show a scatter plot for $\operatorname{Br}(\mathcal{H} \rightarrow \gamma \gamma)$ in the $\left(\lambda_{1}, \lambda_{4}\right)$ plane illustrating more generally the previously discussed behavior, for $m_{\mathcal{H}}=115 \mathrm{GeV}$ (left) and $m_{\mathcal{H}}=127 \mathrm{GeV}$ (right), imposing unitarity and BFB constraints as well as the lower bounds $m_{H^{ \pm}} \gtrsim 80 \mathrm{GeV}$ and $m_{H^{ \pm \pm}} \gtrsim 110 \mathrm{GeV}$ on the (doubly-)charged Higgs masses. One retrieves the gradual enhancement of $\operatorname{Br}(\mathcal{H} \rightarrow \gamma \gamma)$ in the regions with large and positive $\lambda_{1,4}$. The largest region (in yellow) corresponding to $\operatorname{Br}(\mathcal{H} \rightarrow \gamma \gamma) \lesssim 2 \times 10^{-3}$ encompasses three cases: -the SM dominates - complete cancellation between SM and $H^{ \pm}, H^{ \pm \pm}$loops $-H^{ \pm}, H^{ \pm \pm}$loops dominate but still leading to a SM-like branching ratio.

In figures 4, 5 we illustrate the effects directly in terms of the ratio $R_{\gamma \gamma} \approx \sigma^{\gamma \gamma} / \sigma_{\mathrm{SM}}^{\gamma \gamma}$ defined in eq. (4.1), for benchmark Higgs masses. We also show on the plots the present experimental exclusion limits corresponding to these masses, taken from [5]. As can be seen from figure 4 , one can easily accommodate, for $m_{\mathcal{H}} \approx 125 \mathrm{GeV}$, a SM cross-section, $R_{\gamma \gamma}\left(m_{\mathcal{H}}=125 \mathrm{GeV}\right)=1$, or a cross-section in excess of the SM, e.g. $R_{\gamma \gamma}\left(m_{\mathcal{H}}=125 \mathrm{GeV}\right) \sim$ $3-4$, for values of $\lambda_{1}, \lambda_{4}$ within the theoretically allowed region, fulfilling as well the present experimental bound $m_{H^{ \pm}} \gtrsim 80 \mathrm{GeV}$ and the moderate bound $m_{H^{ \pm \pm}} \gtrsim 110 \mathrm{GeV}$ as discussed previously. The excess reported by ATLAS and CMS in the diphoton channel can be readily interpreted in this context. However, one should keep in mind that all other channels remain SM-like, so that the milder excess observed in $W W^{*}$ and $Z Z^{*}$ should disappear with higher statistics in this scenario. This holds independently of which of the two states, $h^{0}$ or $H^{0}$, is playing the role of the SM-like Higgs.

We comment now on another scenario, in case the reported excess around $m_{\mathcal{H}} \approx$ $125 \mathrm{GeV}$ would not stand the future accumulated statistics. Figure 5 shows the $R_{\gamma \gamma}$ ratio corresponding to the case of figure 2 with $m_{\mathcal{H}}$ close to $115 \mathrm{GeV}$. The large deficit for $R_{\gamma \gamma}$ in parts of the $\left(\lambda_{1}, \lambda_{4}\right)$ parameter space opens up an unusual possibility: the exclusion 

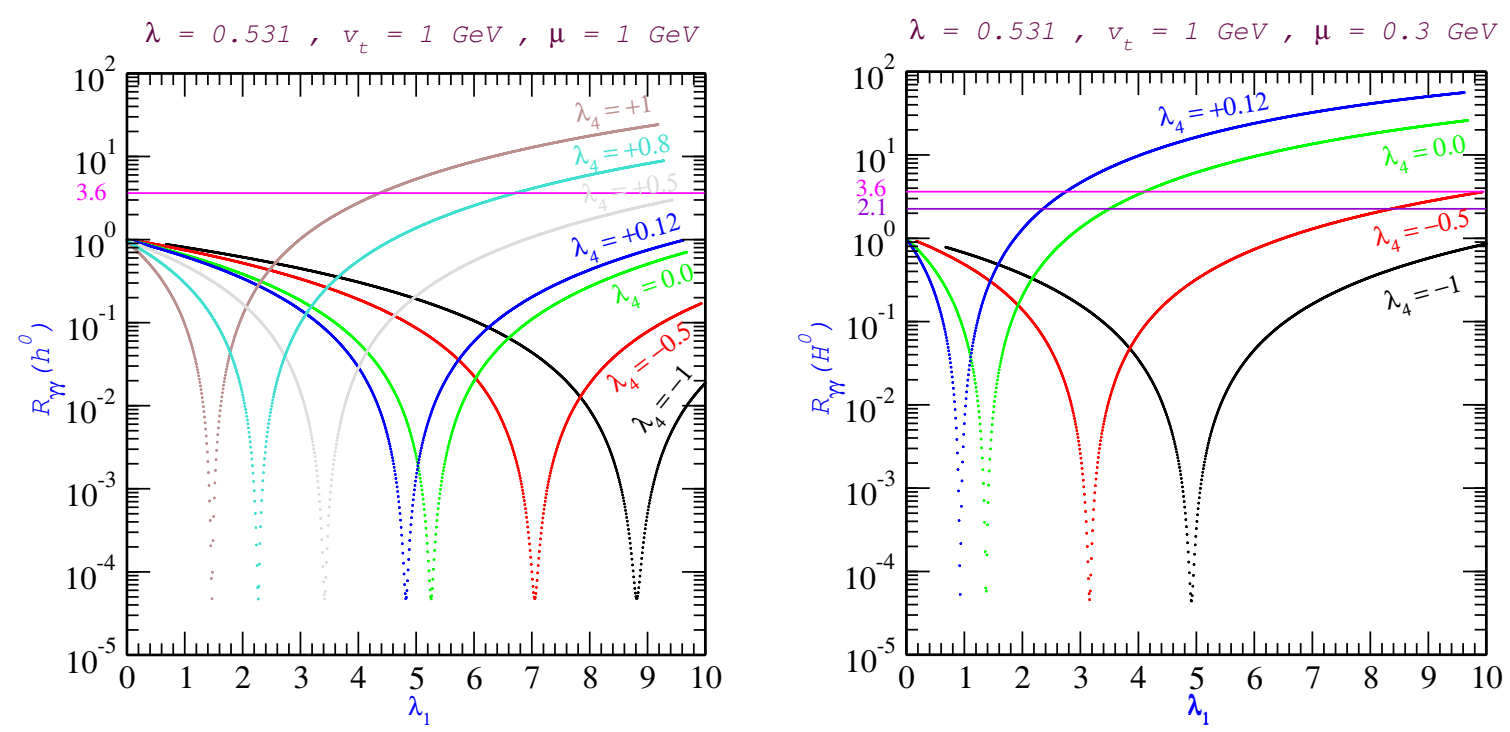

Figure 4. The ratio $R_{\gamma \gamma}$ as a function of $\lambda_{1}$ for various values of $\lambda_{4}$, with $\lambda=0.53, \lambda_{3}=2 \lambda_{2}=0.2$ and $v_{t}=1 \mathrm{GeV}$; left panel: $\mu=1 \mathrm{GeV}, h^{0}$ is SM-like and $m_{h^{0}}=124-125 \mathrm{GeV}$; right panel: $\mu=0.3 \mathrm{GeV}, H^{0}$ is SM-like and $m_{H^{0}}=125-129 \mathrm{GeV}$. The horizontal lines in both panels indicate the ATLAS exclusion limits [5] for $m_{h^{0}}=125 \mathrm{GeV}$ (left) and $m_{H^{0}}=125$ and $129 \mathrm{GeV}$ (right).
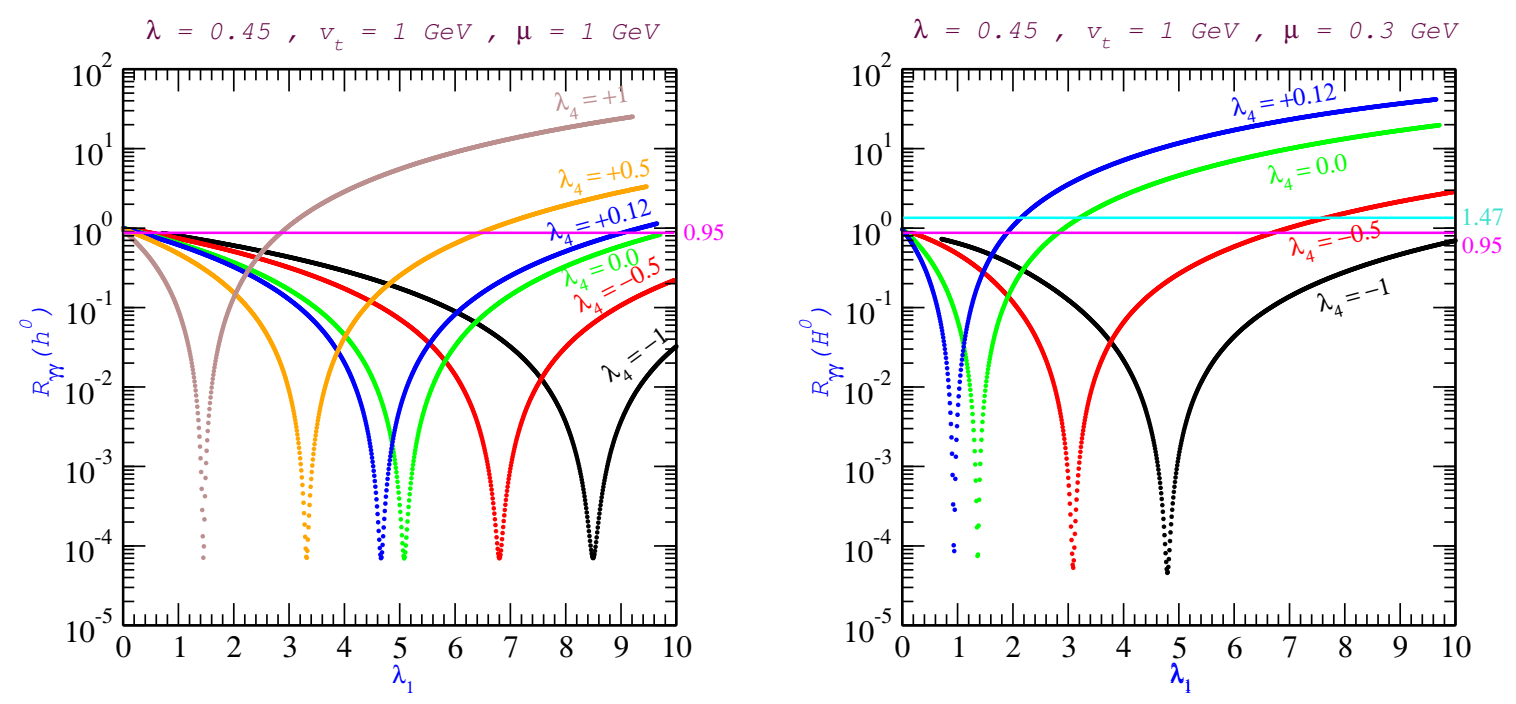

Figure 5. The ratio $R_{\gamma \gamma}$ as a function of $\lambda_{1}$ for various values of $\lambda_{4}$, (other parameters like in figure 2). The horizontal lines in both panels indicate the ATLAS exclusion limits [5] for $m_{h^{0}}=$ $115 \mathrm{GeV}$ (left) and $m_{H^{0}}=115$ and $122.5 \mathrm{GeV}$ (right).

of a SM-like Higgs, such as the one reported by ATLAS in the $114-115 \mathrm{GeV}$ range, does not exclude the LEP events as being real SM-like Higgs events in the same mass range! This is a direct consequence of the fact that in the model we consider, even a tremendous reduction in $\sigma^{\gamma \gamma}=\sigma^{\mathcal{H}} \times \operatorname{Br}(\mathcal{H} \rightarrow \gamma \gamma)$ leaves all other channels, and in particular the LEP relevant cross-section $\sigma\left(e^{+} e^{-} \rightarrow Z \mathcal{H}\right)$ essentially identical to that of the SM.

Last but not least, exclusion limits or a signal in the diphoton channel can be translated 

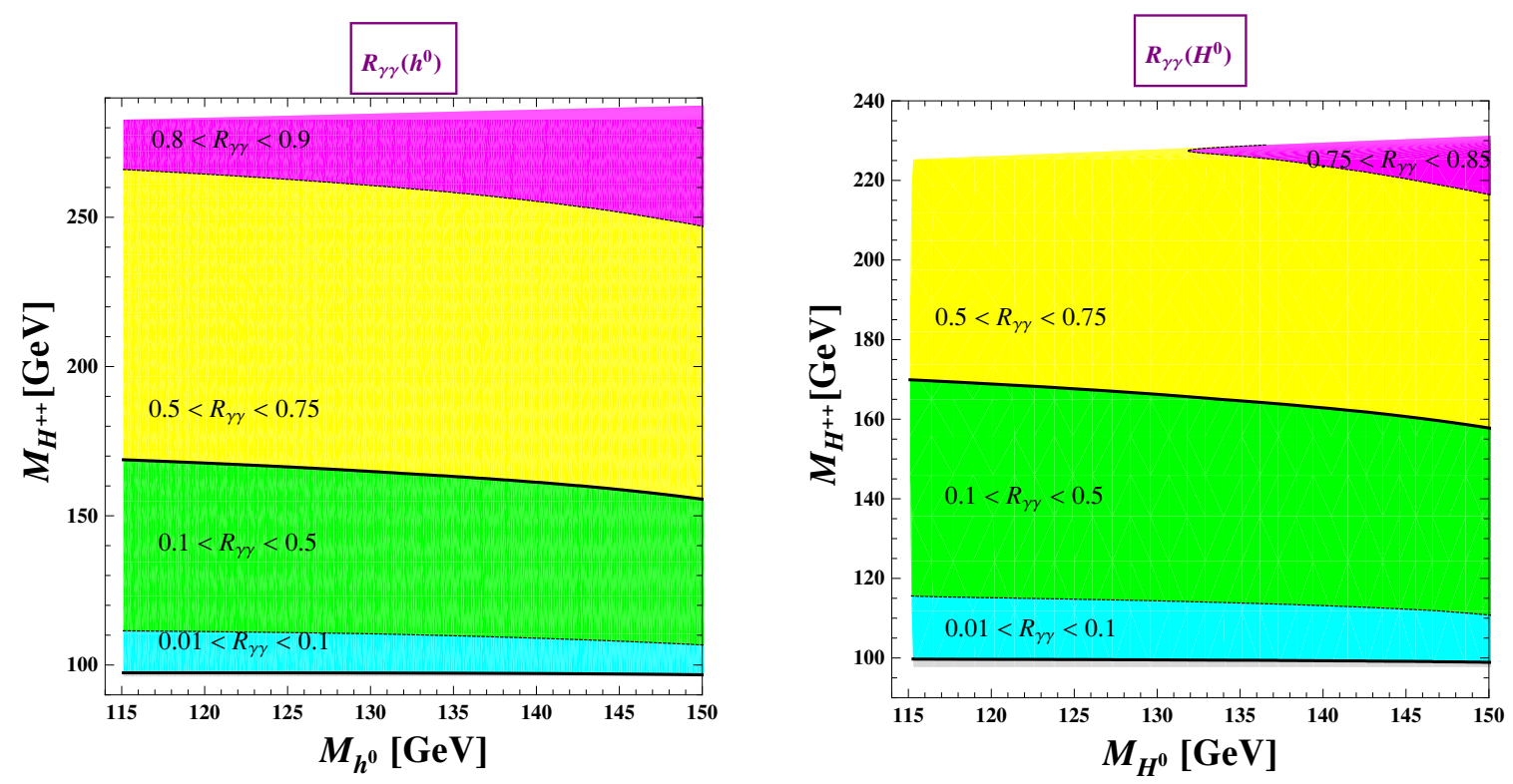

Figure 6. Scatter plots in the $\left(m_{h^{0}}, m_{H^{ \pm \pm}}\right)$and $\left(m_{H^{0}}, m_{H^{ \pm \pm}}\right)$planes, with $h^{0}$ SM-like $(\mu=1 \mathrm{GeV}$, left panel) and $H^{0}$ SM-like ( $\mu=.3 \mathrm{GeV}$, right panel), showing domains of $R_{\gamma \gamma}$ values. We scan in the domain $.45<\lambda<1,-5<\lambda_{4}<3$ with $\lambda_{1}=1, \lambda_{3}=2 \lambda_{2}=0.2$ and $v_{t}=1 \mathrm{GeV}$, consistent with the unitarity and $\mathrm{BFB}$ constraints and requiring $m_{H^{ \pm}} \gtrsim 80 \mathrm{GeV}$.

into constraints on the masses of $H^{ \pm \pm}$and $H^{ \pm}$. We show in figures 6 and 7 the correlation between $m_{\mathcal{H}}$ and $m_{H^{ \pm \pm}}$for different ranges of $R_{\gamma \gamma}$. Obviously, the main dependence on $m_{\mathcal{H}}$ drops out in the ratio $R_{\gamma \gamma}$ whence the almost horizontal bands in the plots. There remains however small correlations which are due to the model-dependent relations between the (doubly-)charged and neutral Higgs masses that can even be magnified in the regime of $H^{0}$ SM-like, albeit in a very small mass region (see bottom panel of 7 ). The sensitivity to the coupling $\lambda_{1}$ can be seen by comparing figures 6 and 7 . For low values of $\lambda_{1}$ as in figure 6 , the ratio $R_{\gamma \gamma}$ remains below 1 even for increasing $H^{ \pm \pm}$and $H^{ \pm}$masses. The reason is that these masses become large when $\lambda_{4}$ is large (and negative) for which the loop contribution of $H^{ \pm}$does not vanish, as can be easily seen from eqs. (2.8), (3.5), (3.7).

In contrast, we see that for the parameter set of figure $7, R_{\gamma \gamma}$ can take SM-like values for $m_{H^{ \pm \pm}}$of order $180 \mathrm{GeV}$, while an excess of 2 to 6 can be achieved for $m_{H^{ \pm \pm}} \approx 130$ $160 \mathrm{GeV}$, and a deficit in $R_{\gamma \gamma}$, down to 2 orders of magnitude, for $m_{H^{ \pm \pm}}$between 200 and $300 \mathrm{GeV}$. Increasing $m_{H^{ \pm \pm}}$(and $m_{H^{ \pm}}$) further, increases $R_{\gamma \gamma}$ again, but rather very slowly towards the SM expectation as can be seen from the upper green region of the plots.

\section{Conclusions}

The very recent ATLAS and CMS exclusion limits for the search for the Higgs boson, clearly indicate that if such a light SM-like state exists, it should be somewhere in the region between 114.4 (LEP) and $130 \mathrm{GeV}$. The diphoton channel is thus expected to play a crucial role in the near future data analyses, eventually confirming the not yet statistically 

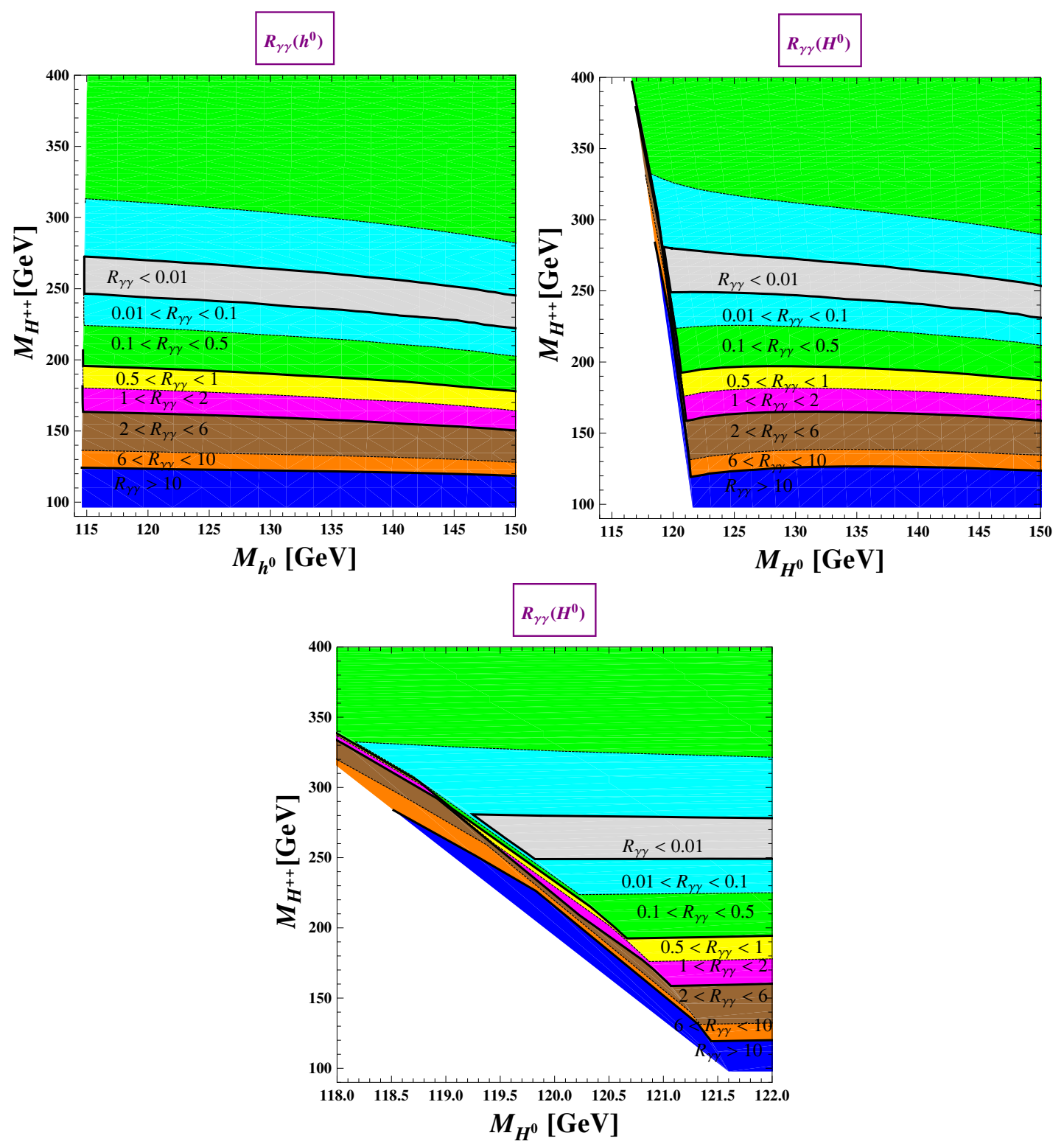

Figure 7. Scatter plots in the $\left(m_{h^{0}}, m_{H^{ \pm \pm}}\right)$and $\left(m_{H^{0}}, m_{H^{ \pm \pm}}\right)$planes, with $h^{0}$ SM-like $(\mu=1 \mathrm{GeV}$, upper left panel) and $H^{0}$ SM-like ( $\mu=.3 \mathrm{GeV}$, upper right panel), showing domains of $R_{\gamma \gamma}$ values. The lower plot zooms on the distinctive features in the $H^{0}$ SM-like case. The scanned domains are as in figure 6 but with $\lambda_{1}=8$.

significant excess around $125 \mathrm{GeV}$. In this paper we have shown that the diphoton channel can be interpreted in a peculiar way in the context of the Type II Seesaw model, provided that the full Higgs sector of the model lies below the $\mathrm{TeV}$ scale. While there is always in this model a neutral Higgs state coupling essentially like the SM Higgs, the diphoton channel can be drastically enhanced or reduced by several factors with respect to the SM prediction, as a result of the loop effects of the doubly-charged (and charged) Higgs states, while all the other relevant decay (as well as production) channels remain at their SM level. 
Theoretically consistent domains of the parameter space in the small $\mu$ regime can thus account either for an excess or for a deficit or even for a SM value of the diphoton crosssection, making the model hard to rule out on the basis of the neutral Higgs observables alone. In particular, the exclusion of a mass region through the diphoton channel does not exclude SM-like Higgs events in the other channels (including the LEP $Z H$ channel) for the same mass region. Rather, it can be re-interpreted in terms of bounds on the masses of the doubly-charged (and charged) Higgs states of the model. The experimental search for such light doubly-charged states through their decay into (off-shell) W bosons is a crucial test of the model while the present bounds based on same-sign di-lepton decays do not necessarily apply in our scenario.

\section{Acknowledgments}

We would like to thank Goran Senjanovic, Dirk Zerwas and Johann Collot for very useful discussions. A.A. and M.C. would like to thank NCTS-Taiwan for partial support, and Chuang-Huan Chen and Hsiang-nan Li for discussions and hospitalty at NCKU and Academia Sinica. This work was supported by Programme Hubert Curien, Volubilis, AI n0: MA/08/186. We also acknowledge the ICTP-IAEA Training Educational Program for partial support, as well as the LIA (International Laboratory for Collider Physics-ILCP).

Open Access. This article is distributed under the terms of the Creative Commons Attribution License which permits any use, distribution and reproduction in any medium, provided the original author(s) and source are credited.

\section{References}

[1] ATLAS collaboration, Combined Standard Model Higgs boson searches with up to $2.3 \mathrm{fb}^{-1}$ of pp collisions at $\sqrt{s}=7 \mathrm{TeV}$ at the LHC, ATLAS-CONF-2011-157 (2011).

[2] ATLAS collaboration, Combination of Higgs Boson Searches with up to $4.9 \mathrm{fb}^{-1}$ of $\mathrm{pp}$ Collisions Data Taken at a center-of-mass energy of $7 \mathrm{TeV}$ with the ATLAS Experiment at the LHC, ATLAS-CONF-2011-163 (2011).

[3] CMS collaboration, Combination of SM Higgs Searches, PAS-HIG-11-032 (2011).

[4] CMS collaboration, Search for a Higgs boson decaying into two photons in the CMS detector, PAS-HIG-11-030 (2011).

[5] ATLAS collaboration, Search for the Standard Model Higgs boson in the diphoton decay channel with $4.9 \mathrm{fb}^{-1}$ of ATLAS data at $\sqrt{s}=7 \mathrm{TeV}$, ATALS-CONF-2011-161 (2011).

[6] W. Konetschny and W. Kummer, Nonconservation of Total Lepton Number with Scalar Bosons, Phys. Lett. B 70 (1977) 433 [inSPIRE].

[7] T. Cheng and L.-F. Li, Neutrino Masses, Mixings and Oscillations in $S U(2) \times U(1)$ Models of Electroweak Interactions, Phys. Rev. D 22 (1980) 2860 [INSPIRE].

[8] G. Lazarides, Q. Shafi and C. Wetterich, Proton Lifetime and Fermion Masses in an SO(10) Model, Nucl. Phys. B 181 (1981) 287 [InSPIRE]. 
[9] J. Schechter and J. Valle, Neutrino Masses in $S U(2) \times U(1)$ Theories, Phys. Rev. D 22 (1980) 2227 [INSPIRE].

[10] R.N. Mohapatra and G. Senjanović, Neutrino Masses and Mixings in Gauge Models with Spontaneous Parity Violation, Phys. Rev. D 23 (1981) 165 [inSPIRE].

[11] A. Akeroyd, M. Aoki and H. Sugiyama, Probing Majorana Phases and Neutrino Mass Spectrum in the Higgs Triplet Model at the CERN LHC, Phys. Rev. D 77 (2008) 075010 [arXiv: 0712.4019] [INSPIRE].

[12] P. Fileviez Perez, T. Han, G.-y. Huang, T. Li and K. Wang, Neutrino Masses and the CERN LHC: Testing Type II Seesaw, Phys. Rev. D 78 (2008) 015018 [arXiv:0805.3536] [InSPIRE].

[13] F. del Aguila and J. Aguilar-Saavedra, Distinguishing seesaw models at LHC with multi-lepton signals, Nucl. Phys. B $\mathbf{8 1 3}$ (2009) 22 [arXiv:0808.2468] [INSPIRE].

[14] A. Akeroyd, M. Aoki and H. Sugiyama, Lepton Flavour Violating Decays $\tau \rightarrow \overline{l l l}$ and $\mu \rightarrow e \gamma$ in the Higgs Triplet Model, Phys. Rev. D 79 (2009) 113010 [arXiv:0904.3640] [InSPIRE].

[15] T. Fukuyama, H. Sugiyama and K. Tsumura, Constraints from muon g-2 and LFV processes in the Higgs Triplet Model, JHEP 03 (2010) 044 [arXiv:0909.4943] [INSPIRE].

[16] A. Akeroyd and C.-W. Chiang, Doubly charged Higgs bosons and three-lepton signatures in the Higgs Triplet Model, Phys. Rev. D 80 (2009) 113010 [arXiv:0909.4419] [INSPIRE].

[17] S. Petcov, H. Sugiyama and Y. Takanishi, Neutrinoless Double Beta Decay and $H^{ \pm \pm} \rightarrow l^{\prime \pm} l^{ \pm}$Decays in the Higgs Triplet Model, Phys. Rev. D 80 (2009) 015005 [arXiv: 0904.0759] [INSPIRE].

[18] T. Fukuyama, H. Sugiyama and K. Tsumura, Phenomenology in the Higgs Triplet Model with the $A_{4}$ Symmetry, Phys. Rev. D 82 (2010) 036004 [arXiv: 1005.5338] [InSPIRE].

[19] A. Akeroyd, C.-W. Chiang and N. Gaur, Leptonic signatures of doubly charged Higgs boson production at the LHC, JHEP 11 (2010) 005 [arXiv:1009.2780] [INSPIRE].

[20] A. Arhrib et al., The Higgs Potential in the Type II Seesaw Model, Phys. Rev. D 84 (2011) 095005 [arXiv: 1105.1925] [INSPIRE].

[21] ATLAS collaboration, G. Aad et al., Limits on the production of the Standard Model Higgs Boson in pp collisions at $\sqrt{s}=7 \mathrm{TeV}$ with the ATLAS detector, Eur. Phys. J. C 71 (2011) 1728 [arXiv: 1106.2748] [INSPIRE].

[22] CMS collaboration, G. Bayatian et al., CMS technical design report, volume II: Physics performance, J. Phys. G 34 (2007) 995 [INSPIRE].

[23] J.R. Ellis, M.K. Gaillard and D.V. Nanopoulos, A Phenomenological Profile of the Higgs Boson, Nucl. Phys. B 106 (1976) 292 [INSPIRE].

[24] B.L. Ioffe and V.A. Khoze, What Can Be Expected from Experiments on Colliding $e^{+} e^{-}$ Beams with e Approximately Equal to $100 \mathrm{GeV}$ ? Sov. J. Part. Nucl. 9 (1978) 50

[25] M.A. Shifman, A. Vainshtein, M. Voloshin and V.I. Zakharov, Low-Energy Theorems for Higgs Boson Couplings to Photons, Sov. J. Nucl. Phys. 30 (1979) 711 [inSPIRE].

[26] A. Djouadi, V. Driesen, W. Hollik and J.I. Illana, The Coupling of the lightest SUSY Higgs boson to two photons in the decoupling regime, Eur. Phys. J. C 1 (1998) 149 [hep-ph/9612362] [INSPIRE].

[27] A. Djouadi, V. Driesen, W. Hollik and A. Kraft, The Higgs photon Z boson coupling revisited, Eur. Phys. J. C 1 (1998) 163 [hep-ph/9701342] [INSPIRE]. 
[28] C.-S. Huang and X.-H. Wu, Damped $\sin (\beta-\alpha)$ of Higgs couplings and the lightest Higgs production at gamma gamma colliders in MSSM, Phys. Rev. D 66 (2002) 075002 [hep-ph/0112356] [INSPIRE].

[29] M. Carena, S. Gori, N.R. Shah and C.E. Wagner, A 125 GeV SM-like Higgs in the MSSM and the $\gamma \gamma$ rate, JHEP 03 (2012) 014 [arXiv:1112.3336] [INSPIRE].

[30] I.F. Ginzburg, M. Krawczyk and P. Osland, Potential of photon collider in resolving SM like scenarios, Nucl. Instrum. Meth. A 472 (2001) 149 [hep-ph/0101229] [INSPIRE].

[31] A. Arhrib, W. Hollik, S. Penaranda and M. Capdequi Peyranere, Higgs decays in the two Higgs doublet model: Large quantum effects in the decoupling regime, Phys. Lett. B 579 (2004) 361 [INSPIRE].

[32] N. Bernal, D. Lopez-Val and J. Solà, Single Higgs-boson production through gamma-gamma scattering within the general 2HDM, Phys. Lett. B 677 (2009) 39 [arXiv:0903.4978] [INSPIRE].

[33] P. Posch, Enhancement of $h \rightarrow \gamma \gamma$ in the Two Higgs Doublet Model Type I, Phys. Lett. B 696 (2011) 447 [arXiv: 1001.1759] [INSPIRE].

[34] D. Lopez-Val and J. Solà, Single Higgs-boson production at a photon-photon collider: general 2HDM versus MSSM, Phys. Lett. B 702 (2011) 246 [arXiv:1106.3226] [INSPIRE].

[35] P. Ferreira, R. Santos, M. Sher and J.P. Silva, Implications of the LHC two-photon signal for two-Higgs-doublet models, arXiv:1112.3277 [INSPIRE].

[36] G. Burdman, C. Haluch and R. Matheus, Is the LHC Observing the Pseudo-scalar State of a Two-Higgs Doublet Model?, arXiv:1112.3961 [INSPIRE].

[37] U. Ellwanger, Enhanced di-photon Higgs signal in the Next-to-Minimal Supersymmetric Standard Model, Phys. Lett. B 698 (2011) 293 [arXiv:1012.1201] [INSPIRE].

[38] S. Moretti and S. Munir, Di-photon Higgs signals at the LHC in the next-to-minimal supersymmetric standard model, Eur. Phys. J. C 47 (2006) 791 [hep-ph/0603085] [INSPIRE].

[39] U. Ellwanger, A Higgs boson near $125 \mathrm{GeV}$ with enhanced di-photon signal in the NMSSM, JHEP 03 (2012) 044 [arXiv: 1112.3548] [INSPIRE].

[40] T. Han, H.E. Logan, B. McElrath and L.-T. Wang, Loop induced decays of the little Higgs: $H \rightarrow g g, \gamma \gamma$, Phys. Lett. B 563 (2003) 191 [Erratum ibid. B 603 (2004) 257-259] [hep-ph/0302188] [INSPIRE].

[41] L. Wang and J.M. Yang, The LHC di-photon Higgs signal predicted by little Higgs models, Phys. Rev. D 84 (2011) 075024 [arXiv:1106.3916] [InSPIRE].

[42] P. Fileviez Perez, H.H. Patel, M. Ramsey-Musolf and K. Wang, Triplet Scalars and Dark Matter at the LHC, Phys. Rev. D 79 (2009) 055024 [arXiv:0811.3957] [INSPIRE].

[43] K. Cheung and T.-C. Yuan, Could the excess seen at 124-126 GeV be due to the Randall-Sundrum Radion?, arXiv:1112.4146 [INSPIRE].

[44] J.F. Gunion, H.E. Haber, G.L. Kane and S. Dawson, The Higgs hunter's guide, Front. Phys. 80 (2000) 1.

[45] M. Spira, QCD effects in Higgs physics, Fortsch. Phys. 46 (1998) 203 [hep-ph/9705337] [INSPIRE].

[46] A. Djouadi, The Anatomy of electro-weak symmetry breaking. II. The Higgs bosons in the minimal supersymmetric model, Phys. Rept. 459 (2008) 1 [hep-ph/0503173] [INSPIRE]. 
[47] T. Hahn, Generating Feynman diagrams and amplitudes with FeynArts 3, Comput. Phys. Commun. 140 (2001) 418 [hep-ph/0012260] [INSPIRE].

[48] T. Hahn and M. Pérez-Victoria, Automatized one loop calculations in four-dimensions and D-dimensions, Comput. Phys. Commun. 118 (1999) 153 [hep-ph/9807565] [InSPIRE].

[49] S. Dittmaier et al, Handbook of LHC Higgs Cross sections: 1. Inclusive Observables, (2011).

[50] See for instance chap. 3 of B. De Wit and J. Smith, Field Theory in Particle Physics, Vol. 1, (1986).

[51] C. Amsler et al, Review of particle physics, Phys. Lett. B 667 (2008) 1.

[52] D0 collaboration, V. Abazov et al., Search for doubly-charged Higgs boson pair production in the decay to $\mu^{+} \mu^{+} \mu^{-} \mu^{-}$in $p \bar{p}$ collisions at $\sqrt{s}=1.96 \mathrm{TeV}$, Phys. Rev. Lett. 93 (2004) 141801 [hep-ex/0404015] [INSPIRE].

[53] D0 collaboration, V. Abazov et al., Search for pair production of doubly-charged Higgs bosons in the $\mathrm{H}^{++} \mathrm{H}^{--} \rightarrow \mu^{+} \mu^{+} \mu^{-} \mu^{-}$final state at D0, Phys. Rev. Lett. 101 (2008) 071803 [arXiv:0803.1534] [INSPIRE].

[54] CDF collaboration, D. Acosta et al., Search for doubly-charged Higgs bosons decaying to dileptons in p $\bar{p}$ collisions at $\sqrt{s}=1.96$ TeV, Phys. Rev. Lett. 93 (2004) 221802 [hep-ex/0406073] [INSPIRE].

[55] The CDF collaboration, T. Aaltonen et al., Search for Doubly Charged Higgs Bosons with Lepton-Flavor-Violating Decays involving Tau Leptons, Phys. Rev. Lett. 101 (2008) 121801 [arXiv: 0808.2161] [INSPIRE].

[56] CMS collaboration, Search for a Higgs boson decaying into two photons in the CMS detector, CMS-PAS-HIG-11-010 (2011).

[57] CMS collaboration, Inclusive search for doubly charged Higgs in leptonic final states at $\sqrt{s}=7 \mathrm{TeV}$, PAS-HIG-11-001 (2011).

[58] J. Garayoa and T. Schwetz, Neutrino mass hierarchy and Majorana CP phases within the Higgs triplet model at the LHC, JHEP 03 (2008) 009 [arXiv:0712.1453] [INSPIRE].

[59] M. Kadastik, M. Raidal and L. Rebane, Direct determination of neutrino mass parameters at future colliders, Phys. Rev. D 77 (2008) 115023 [arXiv:0712.3912] [INSPIRE].

[60] A. Melfo, M. Nemevšek, F. Nesti, G. Senjanović and Y. Zhang, Type II Seesaw at LHC: The Roadmap, arXiv:1108.4416 [inSPIRE].

[61] L3 collaboration, P. Achard et al., Search for charged Higgs bosons at LEP, Phys. Lett. B 575 (2003) 208 [hep-ex/0309056] [INSPIRE].

[62] DELPHI collaboration, J. Abdallah et al., Search for charged Higgs bosons at LEP in general two Higgs doublet models, Eur. Phys. J. C 34 (2004) 399 [hep-ex/0404012] [INSPIRE].

[63] A. Djouadi, J. Kalinowski and P. Zerwas, Two and three-body decay modes of SUSY Higgs particles, Z. Phys. C 70 (1996) 435 [hep-ph/9511342] [INSPIRE].

[64] T.G. Rizzo, Decays of heavy Higgs bosons, Phys. Rev. D 22 (1980) 722 [inSPIRE].

[65] W.-Y. Keung and W.J. Marciano, Higgs scalar decays: $H \rightarrow W^{ \pm}+X$, Phys. Rev. D 30 (1984) 248 [INSPIRE]. 